\title{
Téoros
}

Revue de recherche en tourisme

\section{Dimensions spatiales du tourisme en Gaspésie}

\section{Velitchko Velikov, Jacques Roy et Gilles Brien}

Volume 13, numéro 1, mars 1994

Bas-Saint-Laurent, Gaspésie, Iles-de-la-Madeleine : fascinantes périphéries

URI : https://id.erudit.org/iderudit/1077775ar

DOI : https://doi.org/10.7202/1077775ar

Aller au sommaire du numéro

Éditeur(s)

Université du Québec à Montréal

ISSN

0712-8657 (imprimé)

1923-2705 (numérique)

Découvrir la revue

Citer cet article

Velikov, V., Roy, J. \& Brien, G. (1994). Dimensions spatiales du tourisme en Gaspésie. Téoros, 13(1), 38-39. https://doi.org/10.7202/1077775ar d'utilisation que vous pouvez consulter en ligne.

https://apropos.erudit.org/fr/usagers/politique-dutilisation/ 


\title{
Dimensions spatiales du tourisme en Gaspésie
}

\author{
Velitchko Velikov, Jacques Roy et Gilles Brien"
}

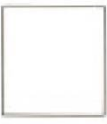

Le *Tour de la Gaspésies est un phénomène touristuque déjà ancien et trè̀s persistant. Près de 500000 personnes parcourent annuellement $1000 \mathrm{~km}$ de routes pendant un peu plus de 5 jours. A l'aide de quelques modèles théoriques, il est intéressant de se pencher sur la structure spatiale de la région touristique gaspésienne. Mais, dans un premier temps, abordons globalement la relation tourisme et espace.

\section{Le phénomène touristique : dimension spatiale}

Les modèles traitant de la dimension spatiale du phénomène touristique ne sont pas très nombreux et ont été, pour la plupart, élaborés sans référence à ceux qui les précédaient; nous pouvons ainsi les présenter sans nous préoccuper de l'ordre chronologique. Toutefois, il s'en dégage une image theorique fragmentée qui est plus difficile à confronter a la réalité.

Miossec (1977) et, plus tard, Lundgren (1982) nous indiquent que par sa nature le produit touristique implique un déplacement du consommateur, une migration vers un espace périphérique. Cela nous permet de distinguer trois types d'espace: espace de résidence, espace parcouru et espace occupé, Gay-Para (1985), quant à lui, désignecesdeux derniers par les termes d'espace regardé et d'espace consommé.

Mais parler de déplacement implique d'aborder, en plus des lieux, les itinéraires employés.

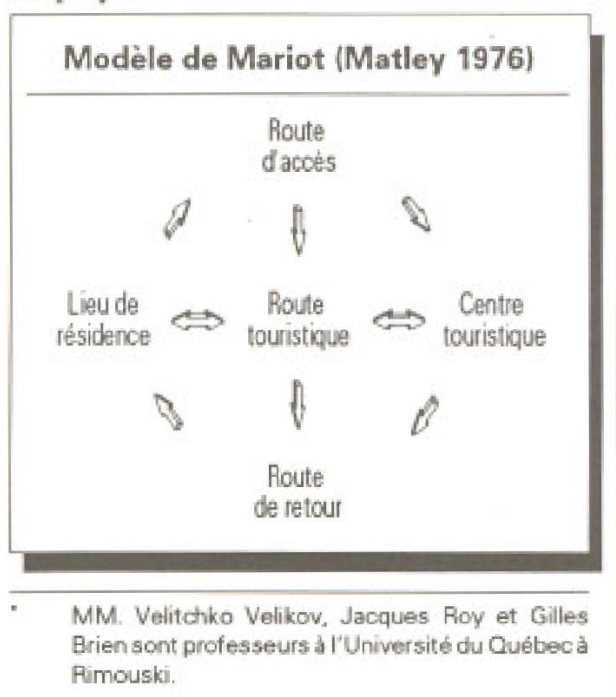

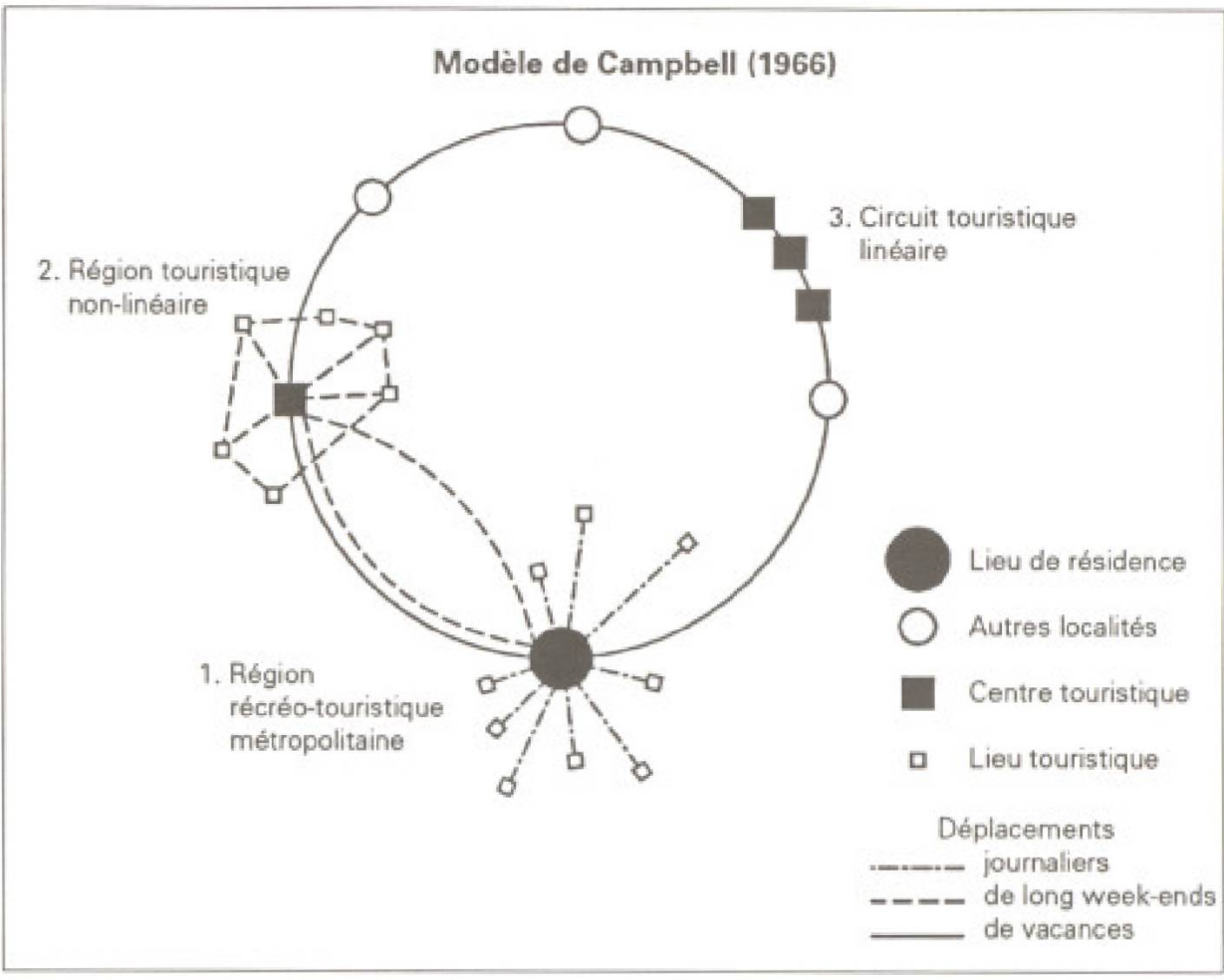

Marriot (1976) nous dit que nous devons pouvoir circonscrire trois types de routes: la route d'acces, la route de retour et la route touristique. La route d'acces constitue un lien direct entre le lieu de résidence et le lieu de séjour. Elle s'oppose à la route touristique oú le produit est directement accessible en cours de déplacement. L'itinéraire de retour constitue l'alternative à la route d'accès; les deux possèdent des caractéristiques similaires.

L'Est du Québec, avec un flux touristique concentré sur la route \# 132, se caractérise par un déplacement liant les trois itinéraires à la fois.

Campbell (1966) nous apporte un troisième élément en faisant le lien entre le déplacement, les activités et la durée du séjour. Ainsi, pour cet auteur, nous pouvons distinguer trois types de zones de réception définis en fonction de l'importance relative du temps de déplacement. II s'agit de la région récréo-touristique métropolitaine, de la région touristique nonlinéaire et du circuit touristique linéaire. La première se caractérise par l'importance de l'activité elle-même, tandis que pour le circuit touristique linéaire, c'est le déplacement lui-même gui prend beaucoup d'importance. La région touristique non-linéaire est une catégorieintermédiaire où nous observons un oomportement à la fois associéà un lieu fixe, aux activités qui y sont pratiquées et à des déplacements intrarégionaux; en région homogène, les efforts de développement privilégient actuellement ce type de zone.

Quant à notre réalité régionale, le modèle de Campbell nous associe au troisième type, c'est-à-dire à une zone de servicesvacances où le déplacement est un aspect importantdu produit touristique. Neparlet-on pas du « Tour de la Gaspésies?

\section{La structure de la zone de réception}

Lelien entrel'importance du déplacement et la structure de la zone de réception a été formalisé par Defert (1966). Selon lui, plus le produit touristique est complexe ou, si vous préférez, plus son degré de finition est élevé, plus la distance que les touristes sont prêts à paroourir est grande.

Miossec (1976) reprend cette idée et l'exprime à l'aide d'un modèle de zones con- 

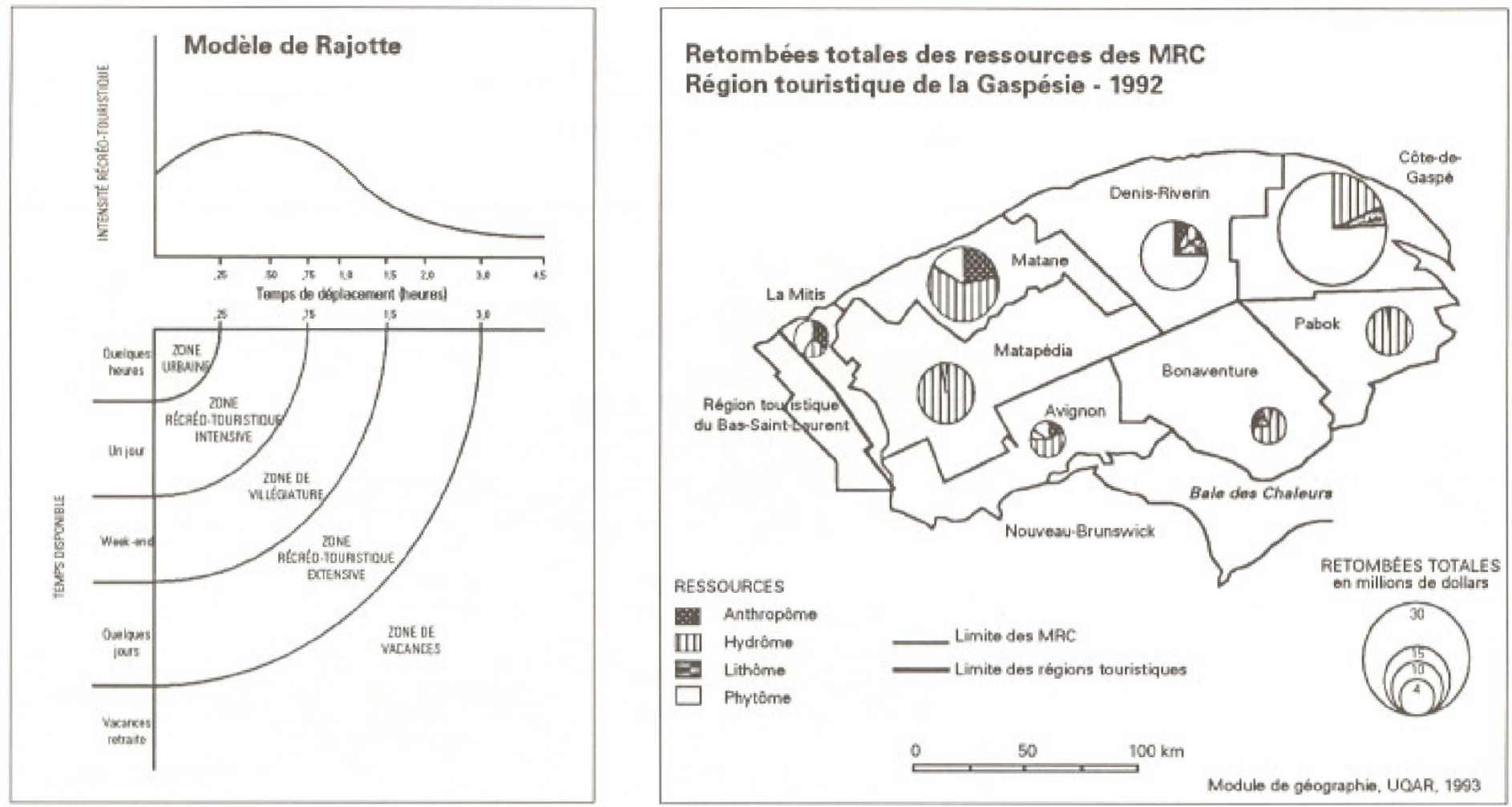

centriques. Bien que mis au point pour expliquer le tourisme international, il peut suggerer ce qui se passe à l'échelle nationale. Ce modèle indique que nous devons nous attendre à distinguer des ceintures, centrées sur les zones émettrices, qui seront déformées en fonction de facteurs tels que les voies de transport, le climat, les monuments historiques, le coût de la vie, etc. Ces premières distorsions causent une altération positive de l'espace touristique, tandis que des facteurs, telle l'instabilité politique, ont un effet négatif.

Rajotte (1975) développe un modèle similaire qu'elle applique a la région de la ville de Québec. Elle observe en effet un comportement où la distance et le produit touristique sont liếs; la première ceinture est essentiellement à vocation récréo-touristique et se situe à moins d'une demiheure de déplacement. La deuxième ceinture est éfalement une zone de récréation, mais ici le déplacement est de l'ordre de quarante-cinq minutes et les activités sont intensives. Du point de vue touristique, ce sont les ceintures trois, quatre et cinq qui sont interressantes. La troisième ceinture correspond à un déplacement d'une fin de semaine età des actuvités de villégiature. $\mathrm{La}$ zone suivante correspond à des déplacements de quelques jours, ou delongs weekends, etelle estcaractérisée pardes activités récréo-touristiques. La cinquième ceinture est la zone de vacances où les distances parcourues sont illimitées; le temps de séjour est de l'ordre des vacances annuelles.
Si nous regardons ce qui se passe dans notre région, nous constatons que le nombre de nuitées estde 5,2 et que le flux est de l'ordre de 400000 , en provenance à $50 \%$ du centre du Québec. Cette réalité contredit donc les modèles.

On constate, en effet, que le type de zone et letemps du parcourssont *théoriquement incompatibles. La Gaspésie demeure un espace parcouru se rapprochant de ce qui se passe en région péri-urbaine lors de longs week-ends.

\section{Réalité et représentation}

Le produit touristique vendu par l'ATRGaspésie peut se résumer aux quatre mots suivants: verdure, soleil, fleuve et mer. On constate donc que cette promotion touristique s'appuie sur une valeur symbolique, par opposition à une valeur d'usage. Dans les faits, on entretient la dichotomie entre ce qui fait l'objet de la promotion et ce qui est consomme par les touristes.

Cette réalité a été mise en évidence par Joseph (1994) qui analyse les retombées économiques directes et indirectes de l'activité touristique gaspésienne. Il a très bien démontré que le produit n'évolue pas et qu'il reste toujours centré sur la chasse et la pêche et sur les pares et réserves. Nous pouvons parler de croissance et non pas de développement du produit.
Du point de vue marketing, la Gaspésie mise actuellement sur la promotion internationale de son produit. Dans l'immédiat, cette approche nous permet de mieux connaître la demande, mais dans un deuxième temps elle nous oblige à revenir au produit et à sa transformation.

Notre réalité touristique est-elle particulière ou les modèles sont-ils globalement faibles? Quelle que soit l'explication, il est clair que l'approche théorique nous permet de dégager certaines constatations et ainsi mieux nous préparer à faire face à l'évolution de ce secteur d'activité. Plus la capacitéd'explication desmodèles sera élevée, plus leur utilité sera grande. $f$

\section{RÉFÉRENCES}

Defert, Pierre, La localisation touristique: problàmes theoriques ot praticues, Berne, Gurten, 1966.

JOSEPH, Dany, L'évolution des prodults ot des activités touristiques doune région restourcé: le cas de la Gaspósie, mémoire de deuxième cycle. Rimouski, UOAR, 1994.

Gay-Para, Guy, La pratique du tourisme, Paris, Ėoonomica, 1985.

Mossec, Jean-Marie, Un modele de Fespece touristique, Études góogr aphiques, no 1, $1977, \mathrm{pp}, 41-48$.

PEA.ACE, Douglas, Tourism Today, Longman Scientific and Technical, 1987.

Pajotte, Freda, A LocstionalAnalysis or Recreational Fachities in the Quebec City Region, Revue de gerographie. XXIX, no 1, Montréal, 1975, pp. 69-74.

YOKENO, Nobumichi, La bealisation de lindustrie tou ristique: application de lanalyse Thinen-Weber, Cahier du tourisme, C9, Adx-en-Provence, 1969. 